\title{
Towards a Structured Online Consultation Tool
}

\author{
Adam Wyner, Katie Atkinson, and Trevor Bench-Capon \\ University of Liverpool, Liverpool, L69 3BX, UK \\ \{azwyner, katie, tbc $\}$ @liverpool.ac.uk \\ http://www.csc.liv.ac.uk/
}

\begin{abstract}
The Structured Online Consultation tool (SCT) is a component tool in the IMPACT Project which is used to construct and present detailed surveys that solicit feedback from the public concerning issues in public policy. The tool is underwritten by a computational model of argumentation, incorporating fine-grained, interconnected argumentation schemes. While the public responds to easy to understand questions, the answers can be assimilated into a structured framework for analytic purposes, supporting automated reasoning about arguments.
\end{abstract}

Keywords: policy-making, online consultation, argumentation.

\section{Introduction}

The Structured Online Consultation tool (SCT) is a component of the IMPACT Project, a European Union funded Framework 7 project in the ICT for Governance and Policy Modeling theme. The focus of the IMPACT Project is to improve public policy-making. In this paper, we first briefly outline the policymaking context of the Project and the other tools. We elaborate on the role of the SCT and introduce Argumentation Schemes. We give an example of how an argumentation scheme, Practical Reasoning, is represented in the SCT as well as how the respondents are led to consider various aspects of the argument, so that specific points of agreement and disagreement can be registered. The tool is underwritten by a computational model of argumentation, which is briefly outlined. Using the model, we can implement a program to automatically reason with respect to arguments that represent defeasible and inconsistent information, to assist in the construction of surveys, and to aggregate, analyse, and evaluate responses to a completed survey. We discuss how argumentation schemes can be analysed in a form compatible with this model of argumentation. As the model is not presented to the respondent, usability is maintained since the respondent need only answer easy to understand, often "yes/no", questions. This version of the SCT is more advanced than previous versions (or related tools) in that it uses richer forms of interconnected, formalised argumentation schemes.

\section{Context and Current Tools}

Policy making is generally viewed as a cyclical, multi-stage process [2]3. Simplifying, we have the following stages: 
- Evaluation: policy analysts look at existing laws and regulations, considering how the laws and regulations achieve the intended goals and identifying conflicts among goals.

- Agenda setting: based on the evaluation, public administrators define areas for change or improvements.

- Policy formulation: given an agenda, policies are proposed and criticised.

- Decision: after consultations about the proposed policies, the draft laws and regulations are introduced into the legislative process.

- Implementation: Once enacted, legislation is enforced.

The IMPACT Project contributes to the policy formulation stage, where proposed laws and regulations are made available for comment to the general public as well as to a selection of stakeholders with a special interest in topics of the policy. Presuming that the policy has been formulated, we can refer to the commenting activity as policy consultation.

There are several current or proposed policy-making support tools in the European Union and the United States which use currently available wiki, comment, email, or social networking technologies (See 7/4 for discussion of other tools such as IBIS+, Compendium, DebateGraph). We discuss several of these briefly in order to set the context for the contribution of the SCT ${ }^{1}$.

The United Kingdom's Cabinet Office Public Reading website2, which currently presents the Protection of Freedoms Bill, uses a website that unfolds the proposed bill, allowing online readers to look at specific sections. At the bottom level, the user can use a threaded comment facility to respond to a particular portion or responses made by other users. With the Public Reading tool, it is difficult to get an overview understanding of the whole policy and the relation of responses to it. Thus, the role and impact of responses is not highlighted. There is no support for analysing the responses, which is then done "manually" by analysts of the consultation, making the contribution of the responses to the development of the policy draft obscure. Moreover, while the responses are specifically linked to parts of the legislation, the unconstrained nature of the responses means the consultation is unstructured and unsystematic. Not only does this allow inappropriate or irrelevant responses, but it may not elicit the kind of important or useful information that is the primary motivation for the consultation in the first place. The Bill itself proposes a solution to some legislative problem; comments on the Bill may discuss alternative solutions. Yet understanding the Bill or alternative solutions may rest on the motivations and justifications underlying the solutions, for example, in terms of social values that the solution promotes. Making these motivations and justifications overt would further support rational analysis and understanding of the Bill, which in turn would better represent the stakeholders' interests and objectives.

The UK Prime Minister's Office ePetition and the European Commission's The European Citizens' Initiative facilities allow citizens to electronically create,

\footnotetext{
${ }^{1}$ All websites accessed March 29, 2011.

2 http://publicreadingstage.cabinetoffice.gov.uk/
} 
sign, and submit petition 3 . By the same token, these tools can be used to "vote" on a policy proposal. The tools, which enable respondents to submit petitions, are web-based versions of what is has been traditionally accomplished manually. Both of these tools contribute to the policy formulation stage of the policymaking cycle, but not to the comment stage. There is no analytic framework. A particular problem is that it is unclear exactly what respondents are signatory to; that is, it provides an unrefined all or nothing representation of a point of view, whereas there may well be respondents who agree with some parts of the proposal, but not other parts, yet nonetheless sign on to the whole. The SCT is designed to differentiate and draw out such subtle alternative viewpoints.

Other initiatives aim to improve the quality of comments to proposed legislation. The US General Services Administration is preparing a tool to support consultation, ExpertNet, which draws "crowdsources" expertise and attempts to structure responses with social networking facilities such as ranking responses, providing specific questions for community voting, or annotating responses, among others. While this does give indicative information on respondents' reactions, the legislation is not represented in an analytic form, much less supporting machine analysis. Rather, the content of the legislation and the reactions to it must be further analysed, though there is no analytic framework. There are additional issues raised about how to identify, certify, and monitor the community of experts. The RegulationRoom is an academically hosted facility for commenting on proposed legislation, providing guidelines on effective comments. This is more substantive than ExpertNet, yet requires highly skilled individuals to follow the guidelines; it may best suit respondents who already participate in policy consultations.

Finally, in the US state of Massachusetts, legislators are using a wiki tool, LexPop, to "crowdsource" the incremental development of legislation 5 . The question here concerns who is in a position to use such a tool, not just in terms of representing the interests of others and reasoning about legislation, which often requires a deep understanding of law and how to author legislation, but also reasoning about legal values and consequences. The success of current wikis (e.g. Wikipedia) rests on an often small coterie of self-selected, self-regulating authors who write about specialist topics, where questions and controversies can be left unresolved and where there are no legislated consequences.

Despite these drawbacks, these current tools and initiatives are clearly potentially important and useful in leveraging current technologies to draw in greater citizen participation to policy-making by making participation easier and improving the informativeness of feedback. However, providing the means to address or avoid these limitations would positively impact on policy making. In particular, the tools discussed above do not further the substantive semantic

\footnotetext{
3 http://petitions.number10.gov.uk/ (archive only) http://ec.europa.eu/dgs/secretariat_general/citizens_initiative/

4 http://expertnet.wikispaces.com/ http://regulationroom.org/

5 http://lexpop.org/
} 
analysis of the comments in a form that supports machine-processing of rich, complex information, particularly where the comments introduce conflicts and inconsistencies that must be reasoned with, that is, they do not make use of current thinking or techniques found in Artificial Intelligence on argumentation.

\section{Contribution of the SCT}

The SCT is based on a formal, computational model of argumentation and argumentation schemes, providing a semantic analysis of the comments in a form which can be processed and reasoned with further. Thus, it contributes what other tools lack. The proposed version of the SCT is more advanced than current tools for it makes use of interconnected, expressive, and formalised argumentation schemes. While it does structure the feedback, it does so in a way that is accessible and corresponds closely with intuitive considerations. Not only is the informativeness of comments increased, but reasoning about conflict and inconsistency is facilitated. Consequently, analysts, policy-makers, and members of the public will have a greater understanding of the meaning and implications of the policy as well as how they might specifically critique or contribute to it. Along with the other tools in the IMPACT toolbox, described below, the SCT provides a means to identify, represent, and reason with information concerning policy, using an underlying computational model.

The SCT is one out of four tools in the IMPACT Project, which is developing a suite of interconnected tools to facilitate public policy deliberations. All the tools share the underlying computational model of argumentation.

- Argument reconstruction, which applies text analytic techniques and tools to source texts and comments on policy.

- Argument visualisation, which provides a graphical representation of elements of the debate concerning the initial policy and responses.

- Policy modeling, which allows users to model alternative outcomes of policies when applied in particular circumstances and the effect on selected cases.

- Structured consultation, which harvests justifications for particular elements of proposed policies in a structured manner.

For the purposes of the IMPACT Project, the four tools, the SCT among them, will be integrated into the IMPACT Project argumentation toolbox, allowing other components of the toolbox to access and exchange common data. The SCT will be a Rich Internet Application (RIA), which are web applications that have many of the characteristics of a desktop application, but are delivered over the internet in a browser, plug-in, sandbox, or virtual machine. In addition, the SCT will be implemented to adhere to the OSGi standard, which provides an environment where applications are modular bundles that are collections of classes, jars, and configuration files that declare their external dependencies and that can be remotely installed, started, stopped, updated, and uninstalled without requiring a system reboot. Finally, as the SCT is to use data from and provide data to other IMPACT Project tools, the SCT will support the 
export and import of argumentation scheme elements in an Extensible Markup Language (XML) format, e.g. the Legal Knowledge Interchange Format (LKIF), via Application Programming Interfaces (APIs). On the project website, one can find further information about the other tools in the toolbox. Underpinning all four of the tools is a formal, computational model of argumentation and policy.

\section{The Representation of Argumentation}

In policy-making, arguments are central since, given the deliberative context of the consultation, contributors respond to some point of the proposed legislation either by arguing for or against that point, or providing alternatives (which may or may not be construed as incompatible). The arguments may take a range of forms such as giving reasons against a point, giving a definition, adding a premise, identifying anomalies, giving a counter-example, or stating conditions under which the rule is inapplicable, among others. While contributors are aware that they are deliberating, they do not usually systematically address issues raised by other contributors, much less formalise the arguments as might a logician so as to enable further reasoning over the responses. By the same token, without some formalisation, further automated processing for reasoning is infeasible. The latter is rather important given the sheer amount and complexity of information users can submit. As most contributors are not trained logicians or computer scientists, they cannot be expected to provide systematic, formal, machine-readable arguments. Given this, we must attempt to bridge the "gap" between the deliberative inputs that the respondents provide and the systematic, formal representations that can be used for further automated processing such as for reasoning. To this end, the IMPACT Project and the SCT use a formal theory of argumentation using Argumentation Schemes.

We briefly outline a formal theory of arguments and argumentation schemes using simplified examples to give a flavour of the main ideas. We initially outline familiar notions of deductive and defeasible arguments. These are related to Argumentation Frameworks (AF), which reason with arguments at an abstract and formal level and which can be implemented and used to calculate sets of consistent arguments. A key problem is to bridge between the arguments that people use and AFs. We address this problem with Argumentation Schemes (AS), which are accessible, prototypical reasoning patterns. After this review, we return to discuss how the SCT uses and presents policy-making arguments.

\subsection{Arguments and Argumentation Frameworks}

Arguments generally are understood as premises followed by a claim; we say that we infer the claim from the premises. In Classical Logic, we can have the following argument, where All men are mortal and Socrates is a man are premises and Socrates is mortal is the claim. 
All men are mortal and Socrates is a man, so Socrates is mortal.

In this deductive argument, where the premises are true, the claim must follow; additional statements would not change this inference.

There are other arguments in which the claim only presumptively or usually follows from the premises; we refer to these as defeasible arguments. For instance, someone may present the following argument (A):

A Nixon was a Quaker, so Nixon was a pacifist.

Others may dispute (A) with (B)-(D), arguing:

B Nixon was a Republican, so Nixon was not a pacifist.

C Nixon was not born in Pennsylvania, so Nixon was not a Quaker.

D Nixon never mentioned Quakerism, so Nixon was not a Quaker.

The claim (B) is contrary to the claim of (A), while claims of $(C)$ and $(D)$ are contrary to the premise of $(\mathrm{A})$.

The arguments we have presented so far we call the level of fully instantiated arguments in the sense that all the predicates (e.g. being a Republican or mentioning Quakerism) and terms (e.g. Nixon or Quaker) are explicitly represented; that is, we have sentences that human users can understand and process in the argument. To automatically process sentences, we need a formal, computational representation of the sentences and of the arguments.

One formal level of representation is provided by Argumentation Frameworks (AF) [5], which have been shown to formally represent and reason with inconsistency and defeasibility. Here we provide some of the intuitive ideas behind AFs. At this level of representation, we abstract from the particular contents of the arguments to view arguments as nodes (this is a move similar to how Classical Propositional Logic abstracts from the particular contents of propositions) and represent the attack relations between the arguments, where attack is derived from a notion of contrariness: since (B) has a contrary of (A), we say that (B) attacks (A); similarly, (C) attacks (A) and (D) attacks (A). In AFs, the attack relation is viewed as an arc between nodes. Given an AF, the arguments and counter-arguments can be represented as a graph. Moreover, conditions can be given for which nodes "survive" attack. For example, since neither $\mathrm{C}$ nor D are attacked, they "survive"; they both attack (A), so (A) is eliminated; (A) and (B) attack one another, but (A) has been eliminated, so (B) "survives". The result is that we have a set of arguments which are consistent, namely $\{(\mathrm{B}),(\mathrm{C})$, (D)\}, which we refer to as an extension. Maximal consistent positions are called preferred extensions of the framework; in a policy-making context, preferred extensions may be understood as policy positions. While in this simple example, AFs may appear as more technically complex than required, where we deal with many nodes in attack relations, the strength of the formalisation, which has been implemented, comes to the fore.

\subsection{Argumentation Schemes}

For the purposes of the SCT, we cannot expect that respondents in the survey are familiar with expressing themselves in terms of deductive or defeasible ar- 
guments, in identifying contrary statements, or in providing ways of attacking arguments. On the other hand, we do not wish to allow entirely unconstrained arguments as discussed in section 2 Yet, the arguments must be understandable to the respondents in the survey. To navigate between the formal and informal yet provide an accessible format for survey respondents, we use argumentation schemes (AS), of which there are many sorts [10].

Here, we consider two ASs, Practical Reasoning (as in 11) and Expert Opinion. Practical Reasoning relates to determining what people should do in a given situation, which is often central to policy-making consultations; Expert Opinion is what is often used to back up or support particular premises of an argument. We outline each of these schemes in terms of two levels, schematic and instantiated, adding further levels in section 6. Other schemes may also be relevant such as Argument from Analogy, or Ad Homenim, among others [10.

Schematically, the Practical Reasoning argumentation scheme is:

- Premise 1a: The current circumstances are R;

- Premise 2a: Doing action A realises goal G;

- Premise 3a: The goal G promotes value V;

- Claim: We should do action A.

The scheme is instantiated when we provide ground terms for the variables $\mathrm{R}$, A, G and V. For example, concerning a policy-making discussion about a ban on fox hunting, the following argument may be put forth 6 :

- Premise 1a: The ban on fox hunting negatively affects the livelihoods of those who make a living from fox hunting;

- Premise 2a: Repealing the ban on fox hunting creates more jobs in the countryside;

- Premise 3a: Creating more jobs in the countryside promotes prosperity.

- Claim: We should repeal the ban on fox hunting.

This may be one of several arguments put forth in favour of this claim.

The Expert Opinion argumentation scheme may be used to argue for or against a particular statement of another scheme, which we give as a template and then as an instantiated example.

- Premise 1b: E is an expert in subject domain $\mathrm{S}$

- Premise 2b: S contains proposition A;

- Premise 3b: E asserts that it is true that A;

- Claim: A

We connect our argumentation schemes - the claim of this Expert Opinion argument is Premise 1a of the previous Practical Reasoning argument. Other premises of the Practical Reasoning argument might also find support from an expert. For illustration, we use made up individuals and domain knowledge.

${ }^{6}$ Derived from an ePetition on fox hunting http://petitions.number10.gov.uk/huntingactrepeal/ 
- Premise 1b: Professor James is an expert on UK rural economic research.

- Premise 2b: UK rural economics research contains the proposition that the ban on fox hunting negatively affects the livelihoods of those who make a living from fox hunting.

- Premise 3b: Professor James asserts that it is true that the ban on fox hunting negatively affects the livelihoods of those who make a living from fox hunting.

- Claim: The ban on fox hunting negatively affects the livelihoods of those who make a living from fox hunting.

Given these arguments, one might be persuaded to repeal the ban on fox hunting. Alternatively, one might object to particular statements within the arguments, thereby denying that the presumptive claim - that the ban on fox hunting should be repealed - follows; such objections are often presented as questions which, if answered negatively, represent objections to a statement in an argument and so imply an attack on the argument. For instance, one might object to Premise 1b, claiming that Professor James is not an expert on UK rural economic research; one might then support this claim by showing that he has not been a member of any professional research organisation for 10 years and has no qualification. Or, one might object to Premise 3a, citing research that jobs which are created in the countryside are so low paying that they are only marginally better than government support, and thereby do not promote prosperity. For each argumentation scheme there are a range of objections (see [10] and [1]). Note in particular, that the argumentation schemes provide clear, fixed, and fine-grained "discussion points", such as those concerning current circumstances, actions, goals, values, expertise, domains, and so on; objections are directed at these points specifically. It is this aspect of argumentation which structures and makes coherent the policy-making. In this way, it returns very specific information to the policy analyst about exactly what respondents object to.

To this point, we have set the context of the SCT and some of the formal technology that it uses. In the next section, we present an overview of a current implementation of the SCT. Then, in the following section, we outline how argumentation schemes are further analysed so as to support fine-grained argumentation about policy-making.

\section{A Prototype Structured Online Survey Tool}

In developing the SCT, the Practical Reasoning argumentation scheme is central, since all policy proposals are based upon a justification of what to do on a specific issue [1]. Moreover, as we have discussed, the Practical Reasoning argumentation scheme is related to and supported by other schemes (e.g. Expert Opinion), which in turn may be supported by still other schemes, thus requiring a network of interrelated schemes. In this section, we discuss elements of a current prototype online application, Parmenides developed in 4, to illustrate some of the interactions between the system and respondent:7; future, richer SCT

\footnotetext{
7 http://www.csc.liv.ac.uk/ parmenides/
} 
implementations, as discussed below, will improve upon this prototype, drawing on evaluation studies from [4]. We indicate the initial presentation of the argument and sample information that leads the respondent through a structured investigation of his views on the proposed policy. While the system is highly structured, it also allows respondents to introduce their own additional information, which can be used in later iterations of the consultative process.

We consider the question of whether or not to repeal the ban on fox hunting in the UK as provided on the Parmenides website. In responding to the survey, the user is led through a series of screens of information, each screen presenting some particular aspect of the debate. The initial screen presents the argument for a particular action as proposed by the government, using the Practical Reasoning scheme of the fox hunting debate above.

Our example is simplified as the implementation allows more complex expression of the current circumstances, a range of goals, and various expressions of the promotion of a value by a goal. For example, rather than one proposition for the statement of current circumstances, Parmenides presents several (based on the ePetition on fox hunting):

The ban gives succor to animal rights extremists; The ban ignores the findings of a government enquiry; The ban prejudices those who enjoy hunting with dogs; Less humane methods of controlling fox population have been introduced; The ban affects the livelihoods of those who make a living from hunting.

The argumentation scheme can have a number of propositions as premises, but there can only be one action that is the claim.

The argumentation scheme gives us a structured presentation of the initial state of the elements of the topic which is being surveyed. This leads to subsequent screens that solicit the respondents' views on particular aspects of the argument. Where a premise is complex, each proposition can be investigated independently. In particular, the respondent is asked questions about each proposition of each portion of the argumentation scheme, choosing whether he agrees, disagrees, or the question is not applicable. By doing so, the respondent indicates precisely what he accepts in connection with the initial argument. For instance, after the initial argument is given, the user will have a screen with some of the following questions about the starting circumstances:

- Does the ban give succor to animal rights extremists?

- Does the ban ignore the findings of a government enquiry?

- Does the ban prejudice those who enjoy hunting with dogs?

- Have less humane methods of controlling fox population been introduced?

- Does the ban affect the livelihoods of those who make a living from hunting?

Answering "yes" endorses a statement from the initial argument, while "no" registers disagreement with the argument on a specific point.

Where the respondent indicates "no", he is led to further screens that examine the justification for the particular proposition and offer the respondent ways to 
object to it, for example, resorting to the Expert Opinion scheme, as discussed above. The survey ends when the respondent has answered all the relevant questions and submitted his answers. At each stage along the way, the respondent has the option to introduce novel elements, which are submitted to the system developers for consideration in future surveys.

In the next section, we discuss theoretical developments to advance the SCT.

\section{Advancing a Structured Online Survey Tool}

To advance the SCT, we must analyse additional argumentation schemes, identify interconnections between them, and decompose them into their fundamental expressions as well as formalise the expressions so we can compute with them. As this is ongoing work, we briefly sketch some of the directions.

\subsection{Additional Argumentation Schemes}

Part of our current research is directed at spelling out additional arguments and their relationships as may be required for policy-making.

Over the course of the presentation of the survey, various statements found in the policy proposal may require additional, appropriate justification. For instance, while the statement concerning livelihoods might be justified by an expert opinion, a statement such as "The ban prejudices those who enjoy hunting with dogs" might instead require justification from survey data since it is not sufficient for one individual to report his opinion on the matter. Similarly, values such as "economic freedom", "animal welfare", or "human rights" may be justified following an Argument from Commitment, for example, citing a government's manifesto in which the government commits to upholding a value. Thus, we must find the relevant set of argumentation schemes for policy-making.

Another aspect of current work is to decompose existing schemes into central and subsidiary schemes. As we have indicated, statements in the policy require different sorts of justifications. One family of justification concerns the sources of information [10] such as Expert Opinion, Position to Know, Citation, Witness Testimony, Perception, Popular Opinion, and others. In our view, such schemes are related to a root argumentation scheme, which we refer to as Argument from Credible Source: $E$ is a credible source in subject domain $S ; S$ contains proposition $A ; E$ asserts that it is true that $A$; therefore, $A$. The idea is that there are different ways to establish the premises using another argument such as Expert Opinion, Position to Know, and so on. In other words, there are levels of justification, giving rise to a tree-like structure.

Another aspect of the decomposition of argumentation schemes is the elaboration of a particular scheme, especially with respect to implicit or presupposed information as indicated by the objections. While some objections are directed at the truth or accuracy of statements given in the initial position, other objections ask whether there are alternatives, for example, Are there alternative ways of realising the same goal? or Does doing the action have a side effect which demotes some other value? For such objections, it is not sufficient simply to state 
that there are alternatives, but that there must be additional justification why those alternatives negatively impact on the initial position.

\subsection{Towards a Formalisation of Argumentation Schemes}

Argumentation schemes can be expressed at several levels of representation, from abstraction to instantiated. We have identified four key levels, partially indicated in section 4.2, each of which is needed to formulate the schemes so we can compute with them, for example, in a logic programming language such as Prolog. Given the focus of this paper and that our analysis is ongoing, we give only a flavour of this work, leaving aside many details, issues, and elaborations.

In section 4.2, we discussed two schemes; here we refer to the Expert Opinion scheme. At the most abstract level, the argument is taken as a whole - there is no differentiation between premises or claim, nor identification of predicates or terms. At the next level, we distinguish premises and the claim, e.g. Premise 1b: Professor James is an expert on UK rural economic research. Note that at this level, the premise is but an unanalysed string. At the next schematic level, we identify the predicate and variable terms in a pseudo-logical form: is-anexpert-on $(E, D)$, where $\mathrm{E}$ is an individual and $\mathrm{D}$ is a domain. Finally, where we substitute variables for instantiated terms, we have an instantiated level: is-anexpert-on(Professor-James, UK-rural-economic-research). We can compute with these different representations.

The other statements in the Expert Opinion scheme are analysed into predicates and terms. For example, inDomain (T, negAffect $(B, L))$, which instantiated, gives us a expression about the negative affect of the ban on fox hunting to livelihoods, which is true in the domain of UK rural economic research; as well, we have $\operatorname{asserts}(E, \operatorname{neg} A f f e c t(B, L))$, which is true where an instantiation of $\mathrm{E}$ asserts the statement about the ban on fox hunting. For the problem domain, additional predicates will be needed. From such predicates, we can construct a knowledge base as well as arguments according to the schemes. For example, Expert Opinion is the rule $h o l d s(A) \leftarrow[\operatorname{expert}(E, D)$, inDomain $(D, A)$, asserts $(E, A)$, not untrustworthy $(E)$, not notCredible $(E, D)]$, meaning that $\mathrm{A}$ holds where an expert in a domain asserts $\mathrm{A}$, unless the expert is untrustworthy or not credible.

Although a range of schemes have been identified [10, research to systematically create formal representations has only recently begun [116.9. In this way, our work furthers research on argumentation as well as e-participation.

\section{Conclusion}

In this paper we have outlined a Structured Online Consultation tool, which is to be used for the policy consultation phase of the policy-making process. We have described current policy-making tools in use in the UK and the USA along with several limitations. Various tools in the IMPACT Project, the SCT among them, are designed to address these limitations using current web technologies and state-of-the-art computational argumentation techniques. The SCT uses formalised argumentation schemes which enable defeasible reasoning such as found 
in policy consultations, where respondents may disagree. A current prototype implementation was outlined. In the final section, we indicated some aspects of our richer, interconnected, and formal analysis of argumentation schemes.

In future work, we will formally specify the argumentation to be used in the SCT, which will then be implemented in the tool. The tool will also be used and tested in "real world" settings by policy-making organisations, comparing and contrasting the existing tools to the SCT along with taking further guidance on developments of tools for policy making.

Acknowledgments. This work was supported by the FP7-ICT-2009-4 Programme, IMPACT Project, Grant Agreement Number 247228. The work represents the views of the authors and not necessarily the project.

\section{References}

1. Atkinson, K., Bench-Capon, T.J.M.: Practical reasoning as presumptive argumentation using action based alternating transition systems. Artificial Intelligence 171(10-15), 855-874 (2007)

2. Bench-Capon, T.J.: Knowledge based systems applied to law: A framework for discussion. In: Bench-Capon, T.J. (ed.) Knowledge Based Systems and Legal Applications, pp. 329-342. Academic Press, London (1991)

3. Birkland, T.A.: An Introduction to the Policy Process: Theories, Concepts, and Models of Public Policy Making. M.E. Sharpe, USA (2010)

4. Cartwright, D.: Digital Decision-Making: Using Computational Argumentation to Support Democratic Processes. Ph.D. thesis, University of Liverpool (2011)

5. Dung, P.M.: On the acceptability of arguments and its fundamental role in nonmonotonic reasoning, logic programming and n-person games. Artificial Intelligence $77(2), 321-358(1995)$

6. Gordon, T., Prakken, H., Walton, D.: The carneades model of argument and burden of proof. Artificial Intelligence 171, 875-896 (2007)

7. Macintosh, A., Gordon, T., Renton, A.: Providing argument support for eparticipation. Journal of Information, Technology \& Politics 6(1), 43-59 (2009)

8. Prakken, H.: An abstract framework for argumentation with structure arguments. Argument and Computation 1(2), 93-124 (2010)

9. Prakken, H.: On the nature of argument schemes. In: Reed, C., Tindale, C. (eds.) Dialectics, Dialogue and Argumentation. An Examination of Douglas Walton's Theories of Reasoning and Argument, pp. 167-185. College Publications, London (2010)

10. Walton, D., Reed, C., Macagno, F.: Argumentation Schemes. Cambridge University Press, Cambridge (2008)

11. Wyner, A., Bench-Capon, T.: Argument schemes for legal case-based reasoning. In: Lodder, A.R., Mommers, L. (eds.) Legal Knowledge and Information Systems, JURIX 2007, pp. 139-149. IOS Press, Amsterdam (2007) 